\title{
Modélisation et caractérisation des joints collés à hautes vitesses de déformation
}

\section{Modeling and characterization of bonded joints at high strain rates}

\author{
B. Bourel, G. Haugou, F. Lauro, R. Deltombe, D. Lesueur et B. Bennani \\ LAMIH, CNRS UMR 8201, UVHC, Le Mont Houy, 59313 Valenciennes Cedex 9, France
}

\begin{abstract}
This paper deals with the modeling of bonded joints for structures subjected to dynamic crash loading. This new model based on a cohesive element takes into account the viscoelastic behavior, the damage and the failure of the adhesive. Due to the strain rate sensitivity, the identification of failure criterion requires experimental tests, up to very high strain rates. A new testing device has then been set up on the Hopkinson bar in order to load the assemblies with high strain rates and with different angles.
\end{abstract}

Résumé. Ce papier traite de la modélisation de joints collés pour les structures soumises à des sollicitations de type crash. Cette nouvelle modélisation basée sur un élément cohésif tient compte du comportement viscoplastique, de l'endommagement ainsi que de la rupture de l'adhésive. Sensible à la vitesse de déformation l'identification du critère de rupture nécessite une base expérimentale allant jusqu'à de très hautes vitesses de déformations. Un nouveau dispositif d'essais a donc été mis en place sur les barres de Hopkinson afin de solliciter des assemblages à haute vitesse et sous différents angles de chargement.

\section{INTRODUCTION}

Afin d'assurer l'assemblage des nouveaux matériaux, notamment dans le secteur automobile où l'allègement des structures est devenu une priorité majeure, de nouvelles techniques telles que le collage sont de plus en plus utilisées. Le dimensionnement de ces assemblages se confronte néanmoins au manque de modèle de comportement de la colle dans la littérature, aux problèmes de caractérisation dynamique, mais aussi aux difficultés liées à la modélisation EF et aux temps de calculs trop élevés.

Un nouvel élément cohésif basé sur une approche de type DCZM (Discret Cohesive Zone model) [1] a récemment été développé dans afin d'assurer à la fois une modélisation réaliste du comportement de la colle et des temps de calculs acceptables [2]. Des essais ont été réalisés en dynamique notamment pour la partie rupture mais uniquement pour des chargements en traction et en cisaillement. Il est donc nécessaire de fournir au modèle des données dynamiques pour des conditions de chargement mixte.

Le but est donc d'enrichir l'élément précédemment développé grâce à une nouvelle base expérimentale complémentant les essais préalablement réalisés (allant jusqu'à $500 \mathrm{~s}^{-1}$ ). Un nouveau dispositif d'essai a ainsi été développé sur barres de Hopkinson afin d'analyser l'effet de la mixité des contraintes sur le comportement et la rupture de l'adhésif à haute vitesse de déformation. Sur le plan numérique, ces essais ont notamment permis de formuler un nouveau critère de plasticité en contrainte équivalente dans l'élément. Ils ont également permis, comme nous le verrons à travers ce papier, d'améliorer la représentativité du critère de rupture à haute vitesse de déformation.

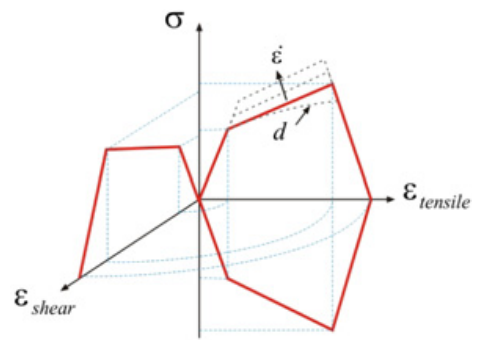

Figure 1. Loi de comportement simplifiée de la colle.

\section{FORMULATION DE L'ÉLÉMENT COHÉSIF}

L'élément cohésif développé dans ces travaux peut être assimilé à un élément ressort généralisé. L'avantage d'une telle modélisation relève des temps de calculs nettement plus faible grâce à un pas de temps de stabilité peu pénalisant pour le schéma d'intégration explicite.

\section{Comportement}

Comparativement à la loi de traction-séparation utilisée par Tvergaard [3] le modèle a été amélioré afin de tenir compte de la viscoplasticité de la colle, de sa différence de comportement en traction et compression, ainsi que de son endommagement et de sa rupture (Fig. 1).

Un critère de plasticité basé sur la contrainte équivalente $\bar{\sigma}$ a été proposé :

$$
f_{n}=\bar{\sigma}-\alpha \sigma_{y} \quad \text { avec } \bar{\sigma}=\sqrt{\left(\alpha \sigma_{n}\right)^{2}+\sigma_{t}^{2}}
$$

où $\sigma_{n}$ et $\sigma_{t}$ sont respectivement les contraintes normales et tangentielles. Le coefficient $\alpha$ exprime la différence de limite élastique entre la traction/compression et le 


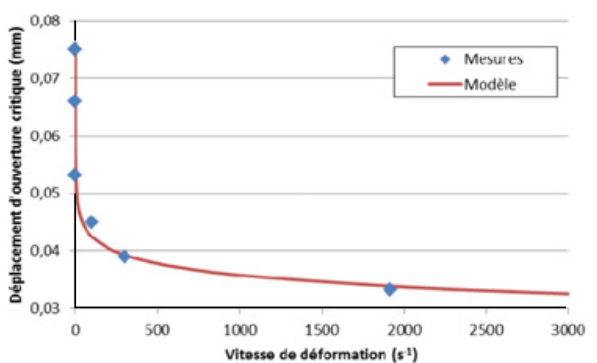

Figure 2. Evolution du déplacement d'ouverture critique.

cisaillement. Enfin, $\sigma_{y}$ est la limite élastique en traction dont l'expression en fonction de la vitesse de déformation suit une évolution du type :

$$
\sigma_{y}=\sigma_{y 0}\left(1+b \log \left(\dot{\bar{\varepsilon}} / \dot{\varepsilon}_{0}\right)\right) .
$$

L'effet de la vitesse de déformation sur la plasticité se traduit par un module tangent visqueux :

$$
E^{T}=c_{0}+c_{1} \dot{\bar{\varepsilon}}+c_{2} \dot{\bar{\varepsilon}}^{2}++c_{3} \dot{\bar{\varepsilon}}^{3} .
$$

Enfin, l'endommagement $d$ est exprimé en fonction de la déformation normal $\varepsilon_{n}$, la croissance et coalescence des cavités dans la colle étant supposées insensibles à la compression et au cisaillement.

$$
d=d_{0}+d_{1}\left(1-e^{d_{2}\left(\varepsilon_{0}-\varepsilon_{n}\right)}\right) .
$$

L'ensemble des coefficients $\sigma_{y_{0}}, b, c_{i}, d_{i}$ et $\varepsilon_{0}$ sont les paramètres matériaux identifiés expérimentalement.

\section{Rupture}

La modélisation de la rupture repose sur un critère de d'initiation, fonction des déplacements d'ouvertures $\delta_{n}$ et de glissements $\delta_{t 1}, \delta_{t 2}$, entre les faces supérieur et inférieur de l'élément. En admettant que la compression n'ait pas d'effet sur la rupture, ce critère peut s'exprimer sous la forme :

$$
\begin{aligned}
& \sqrt{\left(\delta_{t_{1}} / \delta_{t_{1}}^{\text {crit }}\right)^{2}+\left(\delta_{t_{2}} / \delta_{t_{2}}^{\text {crit }}\right)^{2} \geq 1} \text { si } \delta_{n} \leq 0 \\
& \sqrt{\left(\delta_{n} / \delta_{n}^{c r i t}\right)^{2}+\left(\delta_{t_{1}} / \delta_{t_{1}}^{c r i t}\right)^{2}+\left(\delta_{t_{2}} / \delta_{t_{2}}^{c r i t}\right)^{2} \geq 1} \text { si } \delta_{n}>0
\end{aligned}
$$

où $\delta_{i}^{\text {crit }}$ désigne les déplacements critiques à rupture. Les essais dynamiques ont toutefois montré une forte dépendance des déplacements critiques à la vitesse de déformation (Fig. 2). Un modèle mathématique a donc été identifié afin d'exprimer ces déplacements critiques:

$$
\delta_{i}^{\text {crit }}=p_{i}+q_{i} \ln \left(\dot{\varepsilon}_{i}\right) .
$$

Après initiation, la propagation de fissure est engagée dans l'élément grâce à une vitesse de propagation supposée proportionnelle à la vitesse d'ouverture équivalente $V_{e q}$ :

$$
V_{p}=k \cdot V_{e q} \quad \text { avec } V_{e q}=\sqrt{\dot{\delta}_{n}^{2}+\dot{\delta}_{t_{1}}^{2}+\dot{\delta}_{t_{2}}^{2}} .
$$

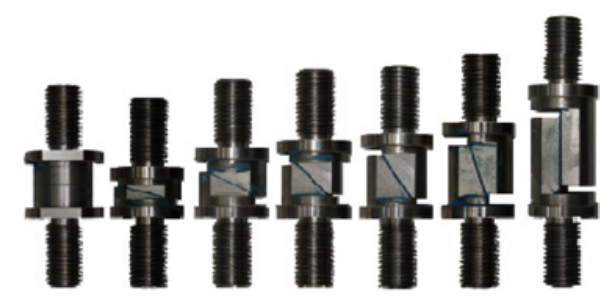

Figure 3. Connecteurs spéciaux.

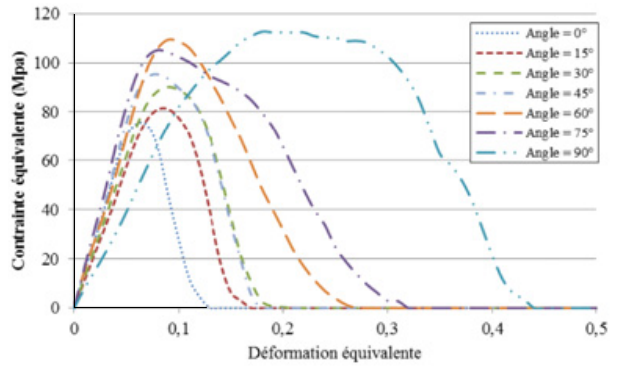

Figure 4. Réponses de la colle aux différents.

\section{ESSAIS SUR ASSEMBLAGES}

La réponse mécanique des joints collés étant fonction de l'angle de chargement et de la vitesse de chargement, un montage particulier a été développé sur un dispositif à barre de Hopkinson en traction. Les barres en acier haute résistance de diamètre $30 \mathrm{~mm}$ sont sollicitées par un impacteur tubulaire en aluminium d'un mètre de long.

Des connecteurs spéciaux assemblés par collage ont été conçus pour être fixé au centre du dispositif entre la barre d'entrée et la barre de sortie. Sept configurations différentes, produisant des angles de chargement compris entre $0^{\circ}$ (traction) et $90^{\circ}$ (cisaillement) ont été testées pour étudier l'effet des contraintes mixtes sur de tels assemblages (Fig. 4).

L'onde traversant le dispositif est mesuré à l'aide de jauges de déformation judicieusement positionnées le long des barres. L'effort $F_{O B}(t)$ transmis par l'assemblage peut ainsi être calculé par :

$$
F_{O B}(t)=S_{O B} \cdot E_{O B} \cdot \varepsilon_{T R A}(t)
$$

où $S_{O B}$ et $E_{O B}$ sont respectivement la section et le module de Young de la barre de sortie. $\varepsilon_{T R A}(t)$ désigne la déformation élastique transmise par l'assemblage et mesurée sur la barre de sortie.

En traction pure le comportement de la colle est quasiment élastique-fragile, avec une rupture qui apparait très rapidement. Une observation des faciès de rupture ne révèle d'ailleurs qu'un très léger blanchissement de la colle. A contrario, le cisaillement fait apparaitre une zone de plasticité nettement plus marqué. Les sollicitations mixtes $\left(15^{\circ}\right.$ à $\left.75^{\circ}\right)$ présentent assez logiquement des comportements repartis à mi-chemin entre la traction et le cisaillement.

\section{CONCLUSION}

Des essais sur assemblages collés ont été réalisés sur barres de Hopkinson en traction afin d'identifier le 
comportement de l'adhésif à haute vitesse de déformation. Ces essais sont venus enrichir la base expérimentale existante $[2,4]$. Ils ont permis d'améliorer le modèle d'élément cohésif développé sous ABAQUS, notamment en introduisant une plasticité équivalente et un critère de rupture sensible à la vitesse de déformation. Des calculs EF portant sur un modèle de barres de Hopkinson sont en cours afin de valider les améliorations apportées à l'élément.

Les présents travaux de recherche ont été soutenus par le CISIT, Campus International sur la Sécurité et l'Intermodalité dans les Transports, la Région Nord-Pas-de-Calais, l'Union Européenne, la Délégations régionales à la Recherche et à la Technologie, le Ministère de l'Enseignement Supérieur et de la Recherche, et le CNRS. Les auteurs tiennent à remercier le soutien de ces institutions.

\section{Références}

[1] Xie D, Waas A. Discrete cohesive zone model for mixed-mode fracture using finite element analysis, Engineering Fracture Mechanics 73 (2006) 17831796.

[2] D. Morin, B. Bourel, B. Bennani, F. Lauro, D. Lesueur. A new cohesive element for structural bonding modelling under dynamic loading, International Journal of Impact Engineering, 53 (2013), 94-105.

[3] Tvergaard, V. and Hutchinson, J.W. (1996b). On the toughness of ductile adhesive joints. J. Mech. Phys. Solids 44, 789-800.

[4] D. Morin, G. Haugou, B. Bennani, F. Lauro. Experimental characterization of a toughened epoxy adhesive under a large range of strain rates. Journal of Adhesion Science and Technology, 25 (2011), 15811602. 